\title{
Make it Your Own: How Process Valence and Self-Construal Affect Evaluation of Self-Made Products
}

\author{
Sukriye Sinem Atakan \\ Ozyegin University \\ Richard P. Bagozzi and Carolyn Yoon \\ University of Michigan
}

\begin{abstract}
Self-production, participation of consumers in the production process of products for their own consumption, leads to consumers' enhanced evaluations of the self-made products. Three experimental studies investigate how and why self-production affects consumers' product evaluations and reveal that not all production experiences create additional value for all consumers. In particular, Studies 1 and 2, using hypothetical stories and real experiences, show that only positive (vs. negative) production experiences enhance evaluations of self-made products over products made by others. Positive (but not negative) experiences decrease the psychological distance between the self and the product and strengthen identification with it. Study 3 manipulates self-construal (independent vs. interdependent) to investigate its role on evaluation of self-made products and products made with close others as a group (i.e., group-made). Consumers with independent self-construal evaluate self-made (vs. other-made) products more favorably only if the process is positive. However, consumers with interdependent self-construal evaluate self-made products more favorably even if the process is negative. Additionally, consumers with interdependent (vs. independent) self-construal exhibit more favorable evaluation of group-made products. Finally, even if consumers know how another person feels while making a product, other people's process emotions do not affect consumers' product judgments as strongly as their own experienced process emotions. ( 2014 Wiley Periodicals, Inc.
\end{abstract}

Modern technology and production methods have enabled consumers to be more involved in the production process of products that they consume (Bendapudi \& Leone, 2003; Prahalad \& Ramaswamy, 2004). Increasingly, companies such as Home Depot, Build-a-Bear, and IKEA encourage consumers to take part in the production process. In addition, many Web sites (e.g., CafePress.com, LapJacks.com, YouBars.com) provide tools for consumers who want to make and purchase selfmade products-products that consumers participate in creating. The range of products that consumers may play a part in creating is extensive. For example, consumers can make designs and place them on anything from mugs to tiles, and from t-shirts to cell phone skins.

Being involved in the creation of a product may generate additional value for consumers and add to quality of life (Xie, Bagozzi, \& Troye, 2008). Experiential (rather than material) products, such as concerts or vacations, have been shown to make individuals happier (Van Boven \& Gilovich, 2003). In addition, investing time rather than money enhances the emotional significance of an event (Mogilner \& Aaker, 2009). Making a product oneself rather than simply buying a finished product, by definition, combines experiential and material aspects of products, and requires an investment of time. Therefore, a consumer's participation in the production process may contribute to happiness and emotional satisfaction derived from consumption behavior, over and above the value placed on the physical product itself. However, there has been limited theoretical speculation and few empirical studies that have focused on self-production, that is, consumers' participation in the production process of products that they consume. The consumer behavior literature has tended to focus on what consumers purchase, an outcome, rather than what they do, a process, in relation to the product (Xie, Bagozzi, \& Troye, 2008).

A number of prior studies in service-dominant logic and cocreation literatures have examined how the integration of consumers into the decision-making mechanisms of a company affects consumer demand (Etgar, 2008; Vargo \& Lusch, 2004), the ownership of the company's products (Fuchs, Prandelli, \& Schreier, 2010), and company attitudes (Fuchs \& Schreier, 2011). The 
focus of these studies has been the interaction of the firm with consumers or consumer groups as part of the firm's business strategy, rather than the personalized relationship between the consumer and the product itself.

Other lines of research that investigate the notions of do-it-yourself (DIY) products, self-production, and prosumption (Toffler, 1980) have recently started to investigate the process of producing goods and services for concurrent or subsequent consumption from the perspective of the consumer. Studies on DIY indicate that consumers may engage in self-production to seek pleasure and self-identity (Williams, 2008; Wolf \& McQuitty, 2011). Moreover, within the context of home meal preparation, Olsen and Mai (2013) show that involvement with food and cooking motivates consumers to participate in the production of meals that they consume and increases time spent preparing and making dinners. Other studies have revealed that participating in the production process of a product enhances the evaluation and value of self-made products (Norton, Mochon, \& Ariely, 2012; Troye \& Supphellen, 2011). In fact, Norton, Mochon, and Ariely (2012) show that consumers find self-made products more valuable than those made by experts, even if the products are quite mundane and utilitarian (i.e., storage boxes, origami animals).

An important question is whether or not all selfproduction creates value for consumers. Imagine that a consumer has just prepared a meal from scratch or assembled a bookcase from IKEA. This person may have gone through a pleasant production process that felt relaxing, soothing, and happy. However, another consumer may have gone through an unpleasant process and felt irritated, annoyed, and unhappy. Would the evaluation of the outcome product be different even if the outcomes from these two processes were identical? Would the process still affect consumers' product evaluations if it were another person going through the unpleasant or pleasant production process? How and why would the production process affect the product evaluations? Does it depend on individual differences? Would how the self is constructed (self-construal; Markus \& Kitayama, 1991a) shape the interpretation of an event that involves the self (i.e., self-production)?

This research investigates the aforementioned questions and focuses on the psychological processes underlying a consumer's interaction with a product during self-production. It examines the conditions and the means by which self-production affects product evaluation. In particular, it looks into the valence of the production process and how a positive versus negative production process affects the person-object relationship and the evaluation of the product. First, it asks, are self-made products evaluated more favorably than other-made products (i.e., made by another person) regardless of whether the process is positive or negative? It investigates whether a person's feelings while making a product affect consumers' evaluation of the outcome product. Then, it studies how self-production cre- ates additional value for consumers and changes the perception of the product. Next, since self-production entails the self, the outcome of this activity is likely to depend on the nature of one's self-representation. Hence, in order to deepen the understanding of consumers' involvement in the production process, this research also investigates how conceptualization of the self (operationalized as independent vs. interdependent self-construal) affects evaluation of self-made products. Finally, in addition to individual self-production conditions, situations when consumers engage in the production process together with close others are also investigated.

The paper begins with a review of the literature on person-object relationship. Then, three experiments explore when and how self-production creates value. The first study uses a hypothetical scenario to test how process valence affects the evaluation of a product made by the individual himself or by another person. The second study also manipulates process valence but requires participants to actually make a product or watch another person go through a similar process. The third study investigates how self-construal affects evaluation of products made by the individual himself or with close others. Finally, the paper discusses how the results add to the understanding of consumers and their relationship with self-made products, implications, and suggestions for future research.

\section{PERSON-OBJECT RELATIONSHIP AND IDENTIFICATION WITH THE PRODUCT}

Theoretical research on "extended self" (Belk, 1988; Pierce, Kostova, \& Dirks, 2003; Sartre, 1943) informs the understanding of how self-production changes the nature of the person-object relationship. Making products oneself is a means of self-extension since the product is imbued with the time, effort, attention, values, and choices of its maker through the process. "Objects such as land to the farmer, handcrafted pieces to the craftsperson, and artworks to the artist may become part of extended-self, because we have intentionally worked upon or created these things, investing both energy and self in them" (Belk, 1988, p. 151). The product is charged with emotional and physical energy of the maker. It starts to reflect the identity of its maker and becomes self-expressive. Investment of self in the object allows individuals to see reflections of themselves in the target object (Mittal, 2006). It symbolizes the individual's identity to the self as well as the outside world. As the object gains a symbolic meaning, the individual becomes psychologically tied to the product.

This paper contributes to the theoretical discussion on extended-self by empirically testing whether participating in the production process of a product affects the person-object relationship. Identification with a focal object (e.g., company, consumer groups, brand) reflects the extent to which the individual sees his or her own 
self-image as overlapping with that of object (Bagozzi $\&$ Dholakia, 2006). Hence, this research proposes that identification with the product, that is, the degree of perceived overlap between one's own current identity or self-image and the product's identity or image as one sees it, reflects at least one dimension of how consumers relate to products. Three experiments test how participating in the production process of a product affects identification with the product and how identification leads to higher evaluations of the self-made product.

\section{HOW DOES PROCESS VALENCE AFFECT PRODUCT IDENTIFICATION?}

Consumer involvement in the production process tends to have a positive effect on product evaluations (Norton, Mochon, \& Ariely, 2012) possibly due to the extension of self to the products that the individual has produced. Troye and Suphellen (2011) mention that when consumers are exposed to something of their own making, they may activate associations with the self and use these associations to form a reaction to the self-made product. Given that people tend to evaluate products associated with the self more favorably (Greenwald \& Banaji, 1995), self-production is likely to result in favorable evaluations of the product. However, in its current form, this logic may be misleading.

Several theorists (Campbell, 1963; Russell \& Mehrabian, 1978) have identified approach and avoidance motivations as playing an integral role in guiding behavior, and recognized valence as the major (although not the only) determinant of approach versus avoidance behavior. Valence has been identified as one of the primary dimensions of emotions (Ekman, 1984; Russell, 1980; Smith \& Ellsworth, 1985). Indeed, the majority of studies on affect and judgment have focused on valence, and shown the influence of valence on satisfaction, judgment, and stereotyping (see Forgas, 2003 for a review).

Previous research (Chen \& Bargh, 1999; Seibt, Neumann, Nussinson, \& Strack, 2007) indicates that positive (vs. negative) valence facilitates approach (vs. avoidance) behavior toward the target object. Positive valence leads to approach tendency that decreases both the physical and psychological distance between oneself and the target. In contrast, negative valence facilitates withdrawal behavior and increases distance between the self and the target. Hence, this research hypothesizes that production process valence will change the psychological distance between the product and the person (identification with the product) and, therefore, affect evaluation of the final product.

An interesting question is whether another maker's process feelings change consumers' evaluation of the outcome product. Process emotions are likely to have more impact on evaluation of the final product when it is the self (vs. another person) who goes through the production process and experiences the emotions since self-relevant information is weighted more heavily than other-related information (Markus, 1977; Weinstein, 1989). Therefore, the following hypotheses are proposed:

H1: The evaluation of a product will be higher when the production process evokes positive rather than negative emotions.

H2: The effect of process emotions on product evaluation will be greater when it is the self (vs. another person) making the product.

H3: To the extent that one participates in the production process, a positive process will increase identification with the product.

H4: The increase in identification with the product explains why self-made products are evaluated more favorably than other-made products.

\section{EFFECT OF PROCESS VALENCE DEPENDS ON SELF-CONSTRUAL}

If an emotional or cognitive activity implicates the self, the outcome of this activity will depend on the nature of one's self-representation. Hence, in order to deepen the understanding of consumers' involvement in the production process, this research also investigates how conceptualization of the self (operationalized as independent vs. interdependent self-construal) affects evaluation of self- and group-made products and the person-object relationship. Self-construal refers to an individual's sense of self in relation to others. Selfconstrual affects the degree to which individuals see themselves as separate from or connected with others. Two primary types of self-construal have been identified: interdependent and independent self-construals (Markus \& Kitayama, 1991a). The interdependent selfconstrual, more common to members of many East Asian and some Latin American and African cultures, defines the self in relation to others. It emphasizes attending to others, fitting in, and promoting social harmony. It takes into account contextual information and synthesizes one's own experience as well as what one believes close others' (family, in-group members) experience in order to understand and shape one's experience (Markus \& Oyserman, 1989). The independent self-construal, more common to members of Western cultures, defines the self in reference to one's own internal emotions and attributes, and others are needed primarily to verify and affirm, rather than to construct, the self (Markus \& Kitayama, 1991a). It focuses on uniqueness, achievement, and autonomy in order to positively differentiate self from others.

Self-construal can influence the nature of an experience, how the experience is constructed, and its consequences. Specifically, this research explores two different ways that self-construal may shape evaluation 
of products when one is involved in production. First, it examines how self-construal affects product evaluations when consumers participate in making products with close others as a group. Second, it investigates how self-construal influences product evaluations when process valence is negative versus positive.

People with interdependent selves tend not to differentiate the self from close others. They are likely to be more attentive and sensitive to close others' experiences than those with independent selves. By contrast, people with independent selves are motivated to separate oneself from others and see the self as different from, and better than, others (Markus \& Kitayama, 1991a). Therefore, it is hypothesized that consumers with interdependent (vs. independent) self-construals who engage in the production process of a product with close others will place greater value on the product.

H5: Consumers with interdependent self-construals will evaluate group-made products more positively than people with independent selfconstruals.

Besides influencing responses to social production situations, self-construal affects people's responses to negative information about the self. People with independent self-construals selectively attend to positive information about the self in order to maintain a positive view of the self (Heine, Kitayama, \& Lehman, 2001; Heine \& Lehman, 1999; Lee, Aaker, \& Gardner, 2000). In fact, strategies to protect a positive self-view, such as decreasing the perceived relevance of a task if one performs poorly on it (Tesser \& Paulhus, 1983) or exhibiting self-enhancement tendencies (Campbell, 1986; Marks, 1984), are quite common among people from North America, but are less common among people from Eastern cultures (Markus \& Kitayama, 1991b).

Although discovery of negative self-related information is threatening and, therefore, avoided by people with independent selves, it is useful for people with interdependent selves and even sought out (Heine, Kitayama, \& Lehman, 2001). Self-related negative information for interdependent selves suggests where adjustments need to be made to better fit group standards, avoid conflict, and maintain social harmony (Heine, Kitayama, \& Lehman, 2001; Lee, Aaker, \& Gardner, 2000). Even if self-related information is negative, it still contributes to one's sense of self and helps one to construct a self-identity. Thus, the interdependentbased (vs. independent) person is less likely to be threatened by and avoid negative self-related information. Negative information threatens the independent self-construal that a person tries to protect. The personal self is not changed but rather maintained when faced with negative self-related information such as shame (Bagozzi, Verbeke, \& Gavino, 2003). Negative information for an interdependent self prompts efforts to improve the self. Instead of avoiding and dissociating the self from it, an interdependent self is likely to use negative information to make judgments and repair relationships.

In fact, when consumers with independent selfconstrual face a threat to an aspect of their identity, they become highly motivated to see themselves in a more positive light and, therefore, avoid products associated with that identity (White \& Argo, 2009). Negative experiences that relate to the self are threatening and hence avoided by independents. On the other hand, when consumers with interdependent self-construal experience a social identity threat, their belongingness needs are activated. Consequently, they are more likely to associate themselves with identity-relevant products and increase their evaluation of the identitylinked product in the face of a threat (White, Argo, \& Sengupta, 2012).

As a result, it is hypothesized that, for consumers with independent self-construals, product evaluation as well as identification with the product are likely to increase only if the self participates in a positive production process since these consumers are likely to dissociate themselves from negative experiences. For consumers with interdependent self-construals, product evaluation, along with identification with the product, is expected to increase regardless of process valence. Consumers with interdependent self-construals may evaluate self-made products (or group-made products where one makes a product with close others) more positively than other-made products even when negative emotions are associated with the production process. More formally, the following hypotheses are proposed:

H6: For consumers with independent selfconstruals, positive (but not negative) process emotions will enhance evaluation of self-made products over other-made products.

H7: For consumers with interdependent selfconstruals, both positive and negative process emotions will enhance evaluations of self-made products over other-made products.

H8: For consumers with independent selfconstruals, positive (but not negative) process emotions will enhance identification with self-made products over other-made products.

H9: For consumers with interdependent selfconstruals, both positive and negative process emotions will enhance identification with selfmade products over other-made products.

To test these hypotheses, three studies are conducted. The first study reveals that even simply imagining the self (vs. another) participating in the production process results in more favorable product evaluations as well as higher identification with the product. The second study extends the findings to an actual 
production context and replicates findings from the first study in a different product domain. It indicates that even a small modification of a product results in identification with the product, and this change in the person-object relationship explains why self-made products are evaluated more favorably than othermade products. The third study examines production situations that involve not only the individual consumer, but also the individual consumer with close others, showing that, compared to people with independent selves, group production processes result in higher evaluation of the final product for people with interdependent selves. Furthermore, results from Study 3 show that negative process emotions do not enhance evaluation of self-made products for people with independent self-construals. However, people with interdependent self-construals evaluate self or group-made products more highly than other-made products, regardless of process valence.

Taken together, the findings suggest that not all self-production experiences enhance product evaluation. When the process is negative, consumers do not distinguish between self-made and other-made products. To the extent that the process is positive, consumers will evaluate self-made products more favorably than other-made products. In addition, another maker's process emotions do not affect a consumer's product evaluation as strongly as experiencing those emotions oneself. Furthermore, the studies address the mechanisms underlying the value creation process through self-production. A positive production experience decreases the psychological distance between the self and the product, and results in extension of the self to the product. Positive self-production processes enhance identification with the product which, in turn, explains why self-made products are evaluated more positively than other-made products. Furthermore, the findings reveal that consumers' self-construal affect their evaluation of products made by themselves or with close others. The three studies identify conditions under which consumers' participation in the production process enhance product evaluations and the mechanisms through which self-production creates value for consumers.

\section{STUDY 1: IMPACT OF PROCESS VALENCE ON PRODUCT EVALUATIONS}

Study 1 tests the basic premise that the valence of production experience affects the evaluation of the final product. Specifically, it investigates the extent to which the effect of process valence on product evaluation depends on whether it is the self or another person who makes the product and goes through the production process. Moreover, it examines whether the hypothesized effect is due to identification with the product.

A 2 (process valence: positive vs. negative) $\times 2$ (maker: self vs. other) between subjects study was de- signed to test the proposed relationships. Throughout all the studies, involvement in making the product is measured to decrease unexplained variance in the dependent variables. Involvement is mainly regarded as personal relevance and motivation to process (Greenwald \& Leavitt, 1984; Petty \& Cacioppo, 1986). Higher involvement levels in making or modifying a product in a given domain may enhance the desire to engage in the relevant behavior (Xie, Bagozzi, \& Ostli, 2013), the process enjoyment, and the likelihood of bonding with the product. However, given that involvement was not a central focus of this paper and has been investigated elsewhere (Olsen \& Mai, 2013), a measure of involvement was included as a covariate in the analyses to control for such effects.

\section{Method}

Participants and Procedure. A total of 172 students (mean age $=20.2,55 \%$ male) completed the study to fulfill course requirements. Participants read a story describing either a pleasant or unpleasant process about making a ceramic mug from clay. They were asked to imagine it was either they or another participant in the experiment making the mug (see Appendix A). In both conditions, a picture of a mug was presented to ensure that negative or positive emotions would not create success or failure perceptions in the process. This provided a conservative test of the hypothesis since the outcome product is held constant across all conditions.

Following the story, all participants reported how much they identify with the product using a 7-point Likert scale ("I would identify with the product"; Pierce, Kostova, \& Dirks, 2003) and a visual diagram of degree of overlap between self-image and product's image (Bergami \& Bagozzi, 2000; $\alpha=0.63$ ). Next, process evaluation (unpleasant/pleasant, unhappy/happy, irritating/soothing, bad/good; $\alpha=0.97$ ) and product evaluation (dislike/like, unpleasant/pleasant, unfavorable/favorable, negative/positive; $\alpha=0.95$ ) were measured. At the end, involvement in making products from clay was measured using Mittal's (1995) modified version of Zaichkowsky's (1985) personal involvement inventory (PII; $\alpha=0.97)$.

\section{Results and Discussion}

Manipulation Check. A 2 (process valence) $\times 2$ (maker) ANCOVA (with involvement as a covariate) on process evaluation yielded a significant main effect of valence $(F(1,167)=430.56, p<0.001)$; the manipulation was successful. Participants in the positive $(M=6.27)$ versus the negative $(M=2.36)$ process condition evaluated the production process more favorably. The main effect of maker was not significant $(F<1)$; however, there was a significant maker $\times$ valence interaction $(F(1,167)=7.57, p<0.01)$. Participants in the "other" condition indicated that the process would be 


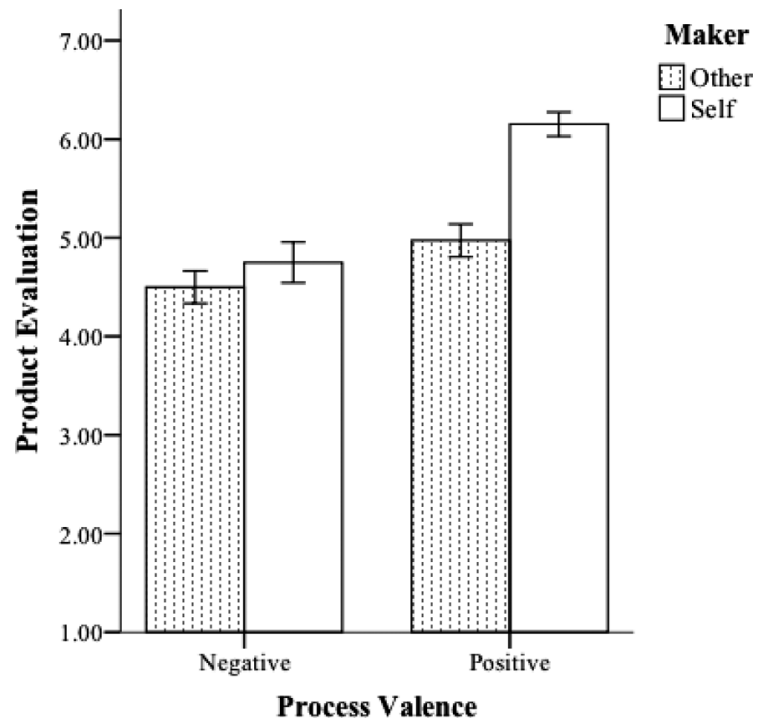

Figure 1. Study 1-Product Evaluation.

less pleasant for the other than for the self in the negative process emotions condition $\left(M_{\text {self }}=2.62, M_{\text {other }}=\right.$ $2.10 ; F(1,167)=4.58, p<0.05)$ and marginally more pleasant for the other than for the self in the positive process condition $\left(M_{\text {self }}=6.02, M_{\text {other }}=6.51 ; F(1,167)=\right.$ $3.17, p=0.08$ ). The covariate (involvement) was significantly related to process evaluation $(F(1,167)=13.44$, $p<0.01$ ). Participants who reported higher levels of involvement in making products from clay indicated more favorable attitudes toward the process.

Tests of Hypotheses. In support of H1, an ANCOVA on product evaluation indicated that the main effect of valence on product evaluation was significant $(F(1,167)$ $=21.66, p<0.001)$. Also, maker $(F(1,167)=18.71, p$ $<0.001)$ and maker $\times$ valence interaction $(F(1,167)=$ $6.25, p<0.01)$ were significant. Simple effects analysis showed that the effect of maker was significant for the positive process condition $\left(M_{\text {self }}=6.08, M_{\text {other }}=4.91\right.$; $F(1,167)=20.80, p<0.001)$, but not for the negative process condition $\left(M_{\text {self }}=4.52, M_{\text {other }}=4.84 ; F(1,167)\right.$ $=1.89, p=0.17$ ). When the process was positive, the self-made product was evaluated more favorably than the other-made product (see Figure 1). In addition, the evaluation of the product did not depend on process valence when another person made the product $(F(1$, $167)=2.14, p=0.14$ ). Participation in the production process was not enough to create value; the process had to be positive to differentially affect product evaluation. Higher levels of involvement in making products from clay (the covariate) enhanced the evaluation of the mug $(F(1,167)=9.43, p<0.01)$.

A key hypothesis concerns the differential impact of the valence of process emotions on product evaluation as a function of maker of the product. H2 suggests that process emotions are likely to affect the evaluation of the product more strongly if it is the self (vs. another person) that experiences the process emotions. This was

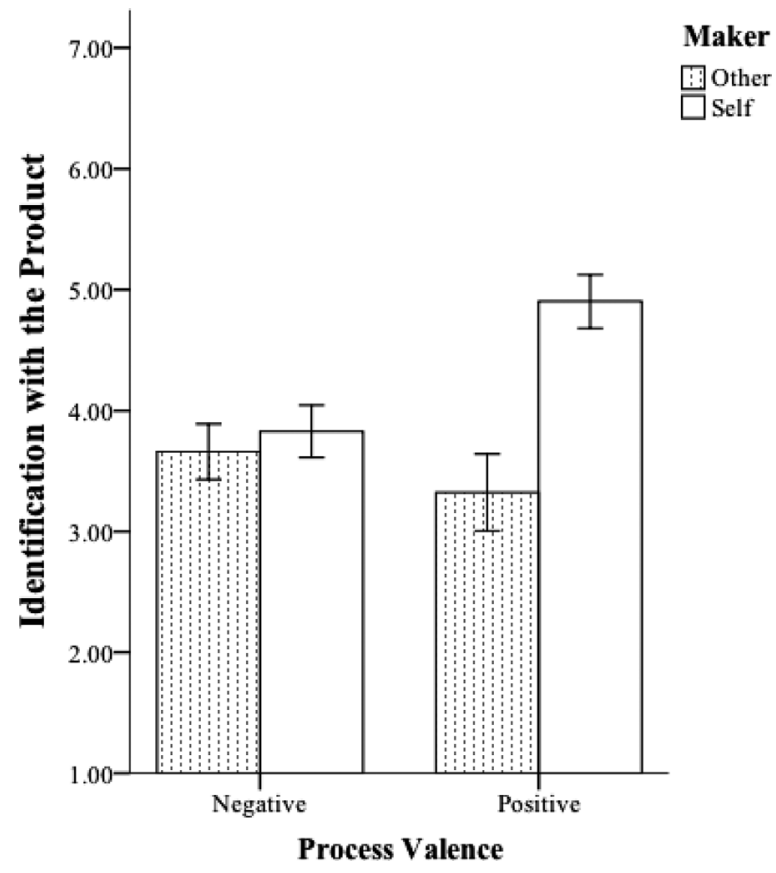

Figure 2. Study 1-Identification with the Product.

tested via a two-way interaction of maker and process evaluation. Because process evaluation is a continuous measure, it was centered for the regression analysis. Regression analysis indicated a significant interaction of maker by process evaluation, thereby supporting $\mathrm{H} 2$ $(\beta=0.29, t=4.16, p<0.01)$. The impact of process emotions on product evaluation was higher when it was the self rather than another person who was involved in the production process.

Identification. Next, identification with the product was analyzed. Analysis indicated a significant effect of maker $(F(1,167=17.85, p<0.001)$ and, consistent with $\mathrm{H} 3$, a maker $\times$ valence interaction $(F(1,167)$ $=7.65, p<0.01$ ). Simple effects tests indicated that making products oneself increased identification with the product in the positive $\left(M_{\text {self }}=4.73, M_{\text {other }}=3.17\right.$; $F(1,167)=21.82, p<0.001)$ but not in the negative $\left(M_{\text {self }}=4.04, M_{\text {other }}=3.71 ; F(1,167)=1.21, p=0.27\right)$ process condition (see Figure 2 ). In addition, when it was another person making the product, process valence did not affect identification with the product ( $p=$ $0.11)$. The covariate, involvement, had a significant effect on identification $(F(1,167)=35.20, p<0.001)$, with higher levels leading to higher identification with the product.

Conditional Indirect Effect. The next question was whether identification with the product mediates the effect of process valence on product evaluation, albeit differently depending on who the maker is (H4). Analysis followed the steps suggested by Hayes (2013) using Model 8 (see Figure 3). Unstandardized identification was used as the mediator and involvement 
the results suggest that even merely imagining making a product can enhance the final evaluation of that product. However, Study 1 used hypothetical stories and required participants to use anticipatory process emotions (rather than actual process emotions) to make product-related evaluations. A question remains as to whether an actual production situation has the same favorable impact on consumers' evaluation of self-made products. Study 2 was conducted to address this question. It was a 2 (process valence: positive vs. negative) $\times 2$ (maker: self vs. other) between subjects design.

\section{Method}

Participants and Procedure. One hundred fortyone students (mean age $=21.2,34 \%$ male) completed the study. The participants were paid $\$ 10$ for their participation. When they arrived at the experimental laboratory, they were given an instruction booklet. The first page introduced the research as a product development study and indicated that the procedure involved designing and painting a white t-shirt. The instructions specified that there should be a geometric figure and a hand-print on the t-shirt (V-neck for females, crew-neck for males).

In the "self" condition, the participants were told that they would paint the t-shirt themselves. In the "other" condition, participants were told that a research assistant would paint the t-shirt for them. Then, all participants filled-out the second page of the booklet containing an order form on which they specified the size of the t-shirt, the geometric figure they wanted on the t-shirt, and the placement of the geometric figure plus the hand-print. The next page provided instructions on how to paint the t-shirt. Finally, the last page had fullsized patterns of geometric figures, one of which they were to use for the t-shirt. Once the participants finished reading and filling out the instruction booklet, they were taken to an individual room where the painting of the t-shirt was done. In the self condition, the room was empty and they were asked to come out once they were done painting the t-shirt. In the other condition, there was a male confederate in the room. The confederate was introduced as a research assistant who would make the t-shirt for them. In reality, he was a senior student at the drama department of the university who was hired and trained to perform the roles described below.

In the positive process condition, the room was orderly; t-shirts were nicely folded and put in boxes according to their sizes; the materials (brush, sponge, towels, cardboard to use during the painting, water) that were used to paint the t-shirt looked neat and clean; there was a chair they could sit on while making the tshirt. In the negative process condition, the room looked disorganized; t-shirts were thrown into a corner and the sizes were mixed together; the materials used to paint the t-shirt looked dirty; the water that was used to clean hands and brushes was blended with paint and olive oil; the table was wobbly; stencil materials were slippery; there was no chair in the room so they had to stand during the process; there was dry paint all over the table, and the instruction booklet had paint and water marks on it (see Appendix B for pictures of the rooms).

In the negative process condition, the actor acted as if the process was unpleasant for him. He did not talk to the participants while he was painting the $t$ shirt; however, he used body language to express he was bored and tired of painting t-shirts, the paint was irritating, the water was smelly and gross, and the wobbly table was annoying. In the positive process condition, both the actor and the participant were seated. The actor acted as if the process was pleasant for him. He enjoyed the process of painting the t-shirt; he liked the feel of the paint; the water was something fun to play with; he smiled most of the time. The actor was trained to follow the same protocol in all sessions. He was instructed to act realistically (not to overact) since the task of painting a t-shirt generally does not create extreme emotions. Once the t-shirt was painted, participants were escorted to a separate area where they filled-out an online survey.

Participants evaluated the process of making the t-shirt using six 7-point semantic differential items (unpleasant/pleasant, unhappy/happy, bad/good, irritating/soothing, unenjoyable/enjoyable, boring/fun; $\alpha=$ 0.96 ). Then, they evaluated the t-shirt ( $\alpha=0.94)$ using the same items from Study 1 and rated themselves (self condition) or the "research assistant" (other condition) in terms of expertise in making $t$ shirts using five 7-point semantic differential items (unskilled/skilled, unqualified/qualified, unknowledgeable/knowledgeable, not an expert/expert, inexperienced/experienced; $\alpha=0.95$ ). In addition, the participants indicated their identification with the product using four 7-point Likert items: "I would identify with the t-shirt," "it would represent who I am," and "it reflects the type of person that I am" were adapted from Reed, Aquino, and Levy (2007); the last item was "the image of the t-shirt fits my self-image" ( $\alpha=0.90)$. The participants used a different scale to report their identification with the product in Study 2 . If the results from Study 1 hold even though the specific measurement of the construct "identification" changes, then it can be concluded that it is identification with the product that drives the results. Finally, involvement in designing clothing items was measured using PII $(\alpha=0.96)$.

\section{Results}

Manipulation Check. An ANCOVA on process evaluation, with involvement included as a covariate, indicated a significant main effect of valence $(F(1,136=$ $7.72, p<0.01$ ); the manipulation was successful. The evaluation of the process was significantly less favorable in the negative $(M=4.31)$ than the positive process condition $(M=4.89)$. There was also a main effect of maker $(F(1,136)=54.19, p<0.001)$. Participants in 
the self condition ( $M=5.36)$ evaluated the process more positively than those in the other condition $(M=3.85)$. The maker $\times$ valence interaction was not significant $(F<1)$. The covariate (involvement) was significantly related to process evaluation $(F(1,136)=15.58, p<$ 0.001 ); participants who reported higher levels of involvement in designing clothing items indicated more favorable attitudes toward the process.

Perceived Expertise. Next, the level of perceived expertise was tested to be the same for the self and other. A 2 (process valence) $\times 2$ (maker) ANCOVA on expertise, with involvement used as a covariate, indicated only a main effect of maker $(F(1,136)=19.85, p<$ $0.001)$. Participants in the other condition $(M=4.43)$ reported higher perceived expertise than did those in the self condition $(M=3.29)$. The participants evaluated the research assistant as having more expertise in painting t-shirts than they did. This may have been due to the fact that it was apparent that the assistant had painted multiple t-shirts during the study; his hands had paint on them when he interacted with the participants. Besides involvement, in order to hold the perceived expertise constant between the self and other conditions, expertise and expertise $\times$ maker interaction (since level of expertise depended on who the maker was) were used as covariates throughout all the subsequent analyses.

Test of Hypotheses. A 2 (process valence) $\times 2$ (maker) ANCOVA on product evaluation (with involvement, expertise, expertise $\times$ maker are used as covariates) indicated, as expected, a significant maker $\times$ valence interaction $(F(1,134)=7.63, p<0.01)$. The covariates, involvement $(F(1,134)=4.77, p<0.05)$, expertise $(F(1$, $134)=18.21, p<0.001)$, and expertise $\times$ maker interaction $(F(1,134)=6.09, p<0.05)$ were significant. Simple effects analysis showed that the maker effect was significant in the positive $\left(M_{\text {self }}=5.69, M_{\text {other }}=\right.$ $4.03 ; F(1,134)=6.68, p<0.05)$ but not in the negative process condition $\left(M_{\text {self }}=4.87, M_{\text {other }}=4.37 ; F<\right.$ 1). Consistent with Study 1 results, only when the process was positive, the self-made product was evaluated more favorably than the other-made product. In addition, the evaluation of the product did not depend on process valence when another person made the product $(F<1)$.

Next, product evaluation was regressed on two-way interaction of maker and centered process emotions to test H2. Replicating findings from Study 1, the maker $\times$ centered process emotions interaction was significant ( $\beta=0.39, t=2.43, p<0.05$ ), thereby indicating the stronger effect of process emotions on product evaluation when self is involved in the production process; $\mathrm{H} 2$ is supported.

Identification. Consistent with $\mathrm{H} 3$, the maker $\times$ valence interaction was significant $(F(1,134)=4.49, p<$ 0.05). Simple effects tests indicated that making products oneself significantly increased identification only when the process was positive $\left(M_{\text {self }}=4.39, M_{\text {other }}=\right.$ $2.88 ; F(1,134)=4.79, p<0.05)$. When the process was negative, there was no difference between evaluation of self-made and other-made products $\left(M_{\text {self }}=\right.$ $\left.3.75, M_{\text {other }}=3.20 ; F<1\right)$. In addition, when it was another person making the product, process valence did not affect identification $(F<1)$. However, as predicted, when the participants painted the t-shirt themselves, positive process did increase identification $(F(1,134)=$ $4.86, p<0.05)$.

Conditional Indirect Effect. Finally, as in Study 1, conditional process analysis was done using bootstrapping (see Table 2 for regression coefficients). In the mediator model, the coefficient for the interaction between process valence and maker was $0.9547(\mathrm{SE}=0.4505$, $\left.C I_{0.95}=0.0637-1.8458\right)$ and statistically different from zero ( $p<0.05$ ). In the outcome model, the effect of identification on evaluation of the product, holding constant both process valence and maker, was 0.3932 ( $S E=$ $0.0732, C I_{0.95}=0.2484-0.5380$ ) and statistically different from zero $(p<0.001)$. The higher the identification, the higher was the evaluation of the product.

Replicating Study 1 findings, the conditional indirect effect of the process valence on product evaluation through identification depended on who the maker was (indirect effect $=0.3754, S E=0.2176, C I_{0.95}=$ $0.0392-0.9079)$. The indirect effect of the process valence on product evaluation through identification was above zero only in the "self" condition (indirect effect $\left.=0.2506, S E=0.1270, C I_{0.95}=0.0481-0.5598\right)$. In the "other" condition, the indirect effect was negative and not different from zero (indirect effect $=-0.1248, S E=$ 0.1596, $C I_{0.95}=-0.5205-0.1310$ ). Thus, identification mediated the effect of the process valence on product evaluation only if it was the self that is going through the production process.

\section{Discussion}

Results from Study 2 replicated Study 1 findings, but in an actual production situation, in a different product category, and with a new measure of identification. The participants were asked to paint a t-shirt themselves or watch another person go through the process of painting a t-shirt. The t-shirt that was painted by the participant himself was evaluated more favorably only when the process was positive. When the process was negative, there was no difference between the tshirt that was painted by another person and the self. It seems that only a positive process creates additional value for self-made products over other-made products.

In addition, as in previous study, Study 2 reveals that how another feels while making a product does not affect one's product judgments as strongly as going through the process oneself. Consumers do not take into account others' process emotions as much as they do their own emotions while making product evaluations. 
Because the proportions of interdependent statements are just the mirror image (i.e., they must add to 1 ) of those for independent statements, the results would have been identical if carried out using those data instead.

Procedure. A similar procedure as in Study 1 was used. The product in the story was a t-shirt. Specifically, participants read either a positive or a negative process story about how they, or another person, or they and their two friends together (group condition) have designed and made a t-shirt (see Appendix C). Then participants evaluated the process using four 7-point semantic differential items (unpleasant/pleasant, unhappy/happy, bad/good, irritating/soothing; $\alpha=0.99$ ) and reported their identification with the product $(\alpha$ $=0.90)$, evaluation of the outcome product $(\alpha=0.96)$, and involvement in designing clothing items $(\alpha=0.96)$ using the same scales from Study 2.

\section{Results and Discussion}

Manipulation Check. An ANCOVA on process evaluation, with involvement included as a covariate, revealed a nonsignificant three-way interaction $(F<1)$ and a nonsignificant main effect of maker $(F(2,233=$ $1.81, p=0.17)$ but significant main effects of valence $(F(1,233=811.72, p<0.01)$, culture $(F(1,233)=20.08$, $p<0.01)$, and two-way interactions of maker $\times$ valence $(F(2,233=13.42, p<0.01)$, maker $\times$ culture $(F(2,233$ $=5.68, p<0.01)$, and valence $\times$ culture $(F(1,233=$ $20.07, p<0.01)$. The process was evaluated to be less pleasant in the negative condition $(M=2.77)$ than it was in the positive condition $(M=6.29)$; the manipulation was successful.

Simple effects indicated that, in the negative, independent self-construal condition $\left(M_{\text {other }}=1.40, M_{\text {self }}=\right.$ $1.52, M_{\text {group }}=1.96$ ) as well as in the positive, interdependent self-construal condition $\left(M_{\text {other }}=6.29, M_{\text {self }}=\right.$ $6.40, M_{\text {group }}=6.25$ ), there were no differences among the other, self, or group conditions in terms of the evaluation of the process (all $p \mathrm{~s}>0.10$ ). In the positive, independent self-construal condition $\left(M_{\text {other }}=6.79, M_{\text {self }}=\right.$ $6.31, M_{\text {group }}=5.72$ ), participants evaluated the process less favorably in the group condition than they did in the other condition $(p<0.05)$. There was no difference between the self and other or self and group conditions (both $p \mathrm{~s}>0.10$ ). On the other hand, in the negative, interdependent condition $\left(M_{\text {other }}=1.93, M_{\text {self }}=3.06\right.$, $M_{\text {group }}=3.79$ ), evaluation of the process was highest in the group condition, followed by the self and other conditions (all $p \mathrm{~s}<0.05$ ). The interdependent self values social interactions and, in fact, uses these interactions to create a sense of self. Hence, it is not unexpected to observe the positive evaluation of the group experience among the participants with interdependent selfconstrual. As expected, the covariate (involvement) was significantly related to process evaluation $(F(1,233)=$ $15.58, p<0.01$ ); participants who reported higher lev- els of involvement in designing clothing items indicated more favorable attitudes toward the process.

Product Evaluation. A 2 (process valence) $\times 3$ (maker) $\times 2$ (self-construal) ANCOVA was conducted on product evaluation, with involvement included as a covariate. As predicted, the analysis yielded a significant three-way interaction $(F(2,233)=3.77, p<0.05)$. Consistent with H5, when the t-shirt was made with close others, product evaluation was higher for Chinese $(M=5.23)$ than for American $(M=4.15)$ participants $(F(1,233)=15.03, p<0.001)$. Making a product with close others generated more favorable product evaluations for people with interdependent than independent self-construals.

As hypothesized (H6) and replicating findings from Studies 1 and 2, planned contrasts revealed that, in the negative process condition, American participants did not change their product evaluation, depending on who the maker was $\left(M_{\text {other }}=3.20, M_{\text {self }}=2.60, M_{\text {group }}=2.67\right.$; $F<1$ ). However, in the positive process condition, the identity of the maker affected the attitudes toward the t-shirt $(F(2,233)=4.18, p<0.05)$; H6 was supported (see Figure 4). As expected, the t-shirt was evaluated more favorably in the self $(M=6.52)$ than in the other $(M=5.31)$ or the group $(M=5.63)$ conditions (both $p \mathrm{~s}<$ $0.05)$. The difference between group and other condition was not significant $(p=0.46)$.

As predicted by H7, when Chinese participants (i.e., those with interdependent self-construal) evaluated the t-shirt, the identity of the maker was significant in the negative process condition $(F(2,233)=7.82, p<0.01)$. Evaluation of the t-shirt was significantly higher in the group $(M=4.08 ; p<0.001)$ and $\operatorname{self}(M=3.60 ; p<0.05)$ conditions than it was in the other condition $(M=2.83)$ when the process was negative. The difference between self and group conditions was not significant ( $p=0.16$ ). In the positive process condition, although the results were in the expected direction, the identity of the maker did not significantly affect the evaluation of the t-shirt $(F(2,233)=1.59, p=0.21)$. Product evaluation in the group condition ( $M=6.38)$ was marginally higher than in the other condition $(M=5.75 ; p=0.08)$, but there was no difference between the self $(M=6.01)$ and group or other conditions (both $p \mathrm{~s}>0.10$ ). This marginal finding might have been due to ceiling effects. Self and group conditions may have been limited by the highest rating of 7 on the scale. Thus, H7 was supported in the negative valence condition, but only marginally supported in the positive valence condition.

Identification. As in the preceding studies, ANCOVA on identification yielded significant main effects for maker $(F(2,233)=32.48, p<0.001)$, process valence $(F(1,233)=26.63, p<0.001)$, self-construal $(F(1,233)$ $=15.56, p<0.001)$, involvement $(F(1,233)=15.17, p$ $<0.001$ ), and a marginal effect for the three-way interaction $(F(2,233)=2.58, p=0.08$; all other $p \mathrm{~s}>0.10)$.

For American (i.e., independent) participants, contrasts revealed that when the process was negative, 


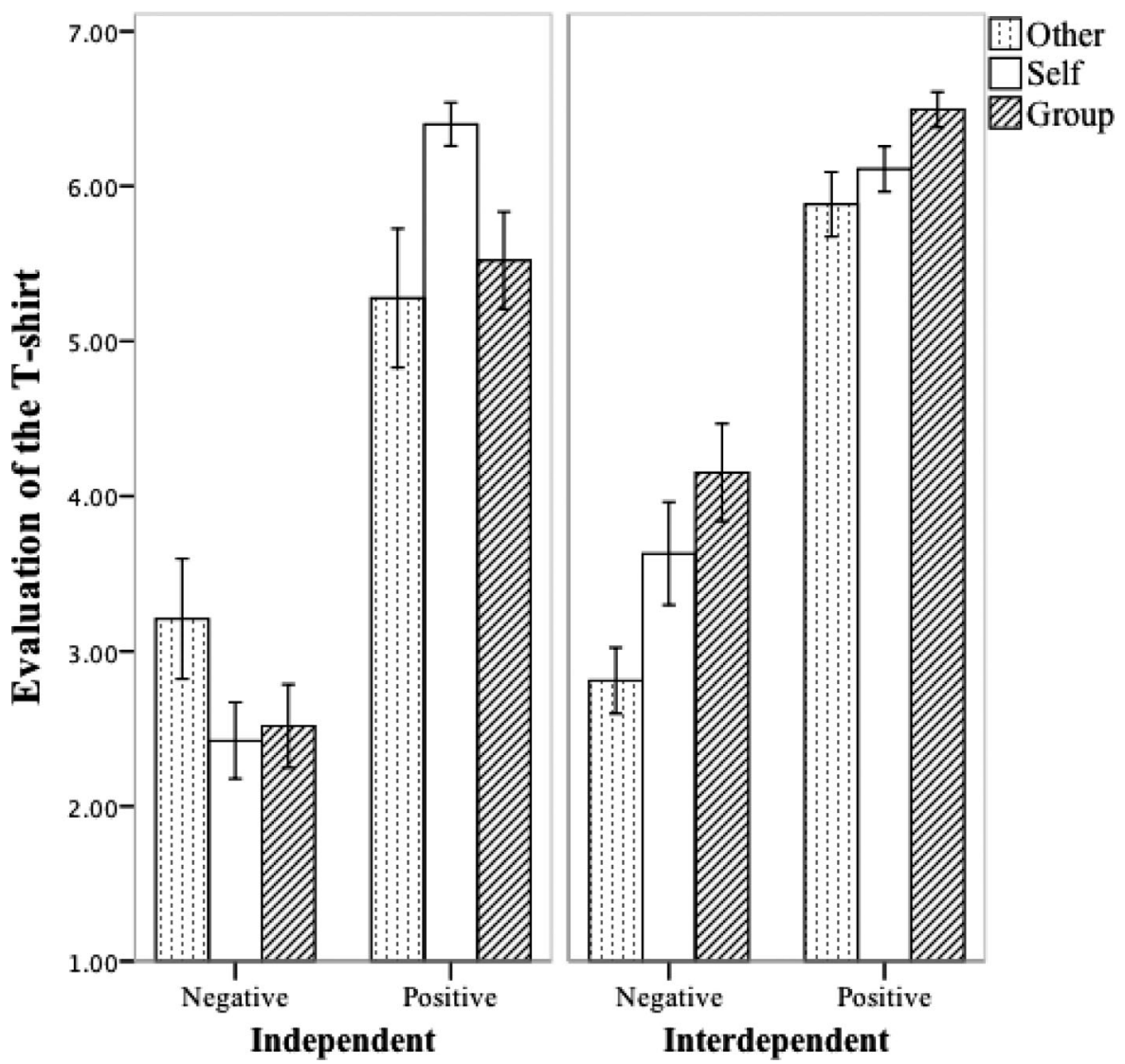

Figure 4. Study 3-Product Evaluation.

identification did not depend on the maker $\left(M_{\text {self }}=\right.$ $4.09, M_{\text {group }}=3.71, M_{\text {other }}=3.11 ; F(2,233)=1.77$, $p=0.17)$. Only the self condition was marginally different than other condition ( $p=0.06$; all other $p \mathrm{~s}>0.10)$. However, when the process was positive, the identity of the maker affected identification $\left(M_{\text {self }}=5.64, M_{\text {group }}=\right.$ $\left.4.41, M_{\text {other }}=3.11 ; F(2,233)=18.52, p<0.001\right)$. Identification with the product was higher in the self than it was in the group condition which was also significantly higher than product identification in the other condition (all $p \mathrm{~s}<0.01$ ). Thus, H8 was supported (see Figure 5).

It was hypothesized that, for Chinese participants, identification would increase regardless of process valence. Contrasts indicated that in both the negative $\left(M_{\text {self }}=4.75, M_{\text {group }}=4.69, M_{\text {other }}=3.09 ; F(2,233)\right.$ $=19.46, p<0.001)$ and positive $\left(M_{\text {self }}=5.72, M_{\text {group }}=\right.$ $\left.5.30, M_{\text {other }}=4.34 ; F(2,233)=7.30, p<0.01\right)$ process valence conditions, product identification in the self and group conditions were not statistically different from each other (both $p \mathrm{~s}>0.10$ ) but were both higher than product identification in the other condition (all $p \mathrm{~s}<$ 0.01). Hence, H9 was supported.

Study 3 indicated that self-construal affects the value derived from participating in the production process in two different aspects. First, self-construal affects product evaluation in a social production context.
Results indicate that making products with close others as a group results in higher product evaluations for people with interdependent (rather than independent) self-construals. Second, self-construal moderates the effect of process valence on product evaluation as well as identification. People with independent self-construals do not evaluate self-made products more positively than other-made products when the process is negative. Only a positive production process generates enhanced product evaluation via greater identification. However, for people with interdependent self-construals, being involved in the production process individually or with close others results in enhanced product evaluation as well as stronger identification, even when the process is negative. This is consistent with prior findings from cross-cultural research that indicates that negative self-related information is threatening, and therefore avoided by independent selves, whereas it is part of a sense of self for interdependent selves (Markus \& Kitayama, 1991b).

\section{GENERAL DISCUSSION}

Hypothetical scenarios as well as an actual production situation have been used in order to understand when and how consumers' participation in the production 


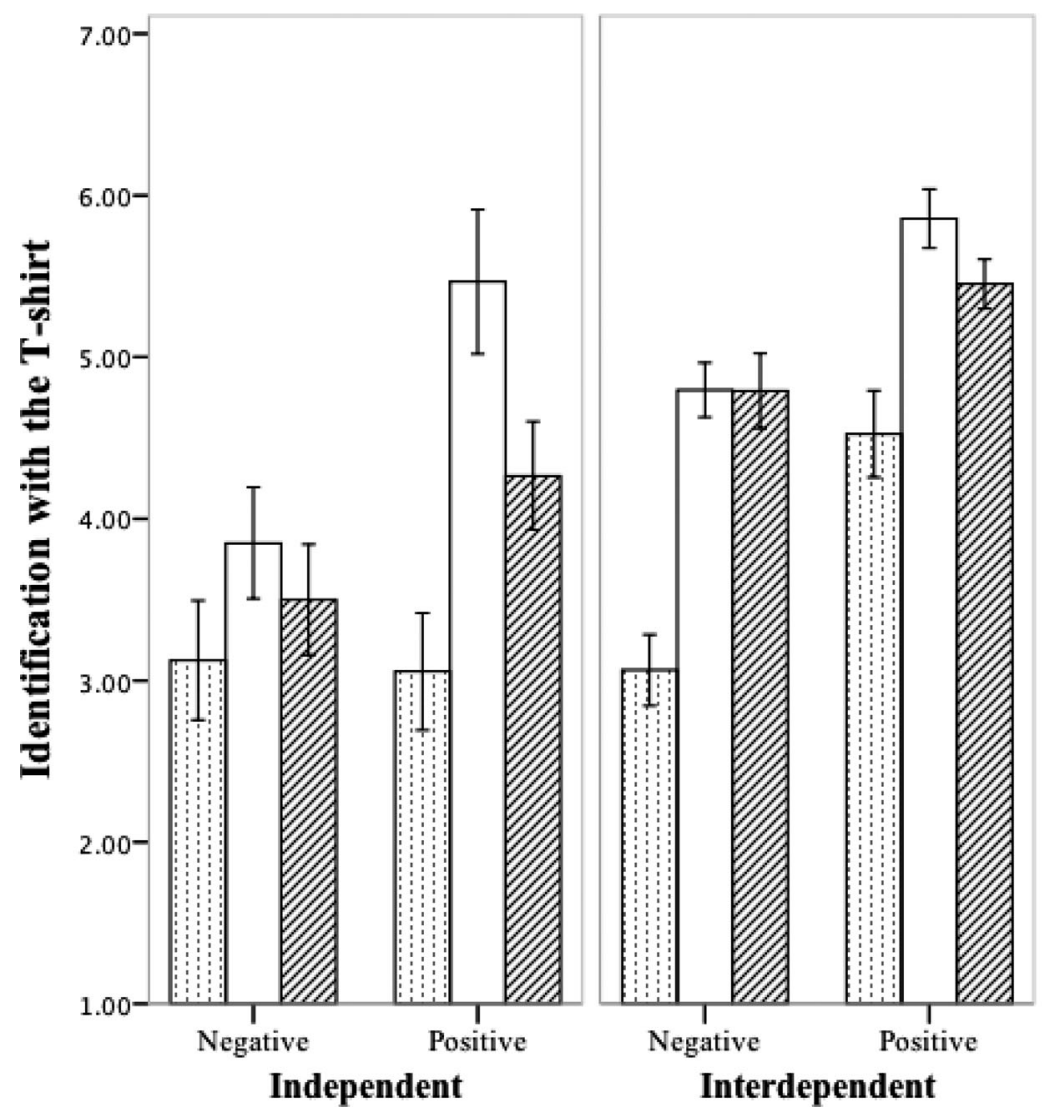

Other
$\square$ Self
ØGroup

Figure 5. Study 3-Identification with the Product.

process creates value. Findings show that even when consumers imagine themselves participating in the production process or when they are minimally involved in a modification of a product, evaluation of the self-made product increases. Moreover, this research empirically investigates how making or modifying a product changes the person-object relationship. Findings reveal that the valence of the emotions that are evoked during the production process affects identification with the product and, therefore, the evaluation of the outcome product when self or self with close others (i.e., group) are involved in the production process.

In addition, although consumers can readily recognize the emotions of another person who makes the product, the influence of process emotions on product evaluation is higher when one's own self is involved in the production. The differential effect of self (vs. another) on product evaluation is driven by heightened identification with the self-made product.

However, under certain conditions (i.e., negative process emotions) and for certain consumers (i.e., those with independent self-construals), being involved in the production process does not produce enhanced product evaluations. A self-made product is not evaluated more positively than an other-made product, when consumers with independent selves go through a negative production process. Independent selves are motivated to positively differentiate self from others, and therefore negative self-information is threatening and, thus, avoided or suppressed. Hence, only when the process is positive does participation in the production process differentiate the evaluation of a self-made from othermade product for consumers with independent selfconstruals. In contrast, consumers with interdependent self-construals evaluate self-made products more positively than other-made products, even when the process is negative. Interdependent selves are not as threatened by negative self-related information as are independent selves, since negative information helps them decide how to modify and construct one's sense of self.

Moreover, the studies show that responses of consumers in social production situations change depending on self-construal. Participating in the production process with close others is likely to affect evaluation by consumers with interdependent self-construals more positively than independent self-construals because the connection of group members to the self is stronger for those with interdependent self-construals.

The findings complement recently emerging research on self-production that has found that participation of consumers in the production process of products influences product evaluations positively (Norton, Mochon, \& Ariely, 2012; Troye \& Supphellen, 2011). Results extend and deepen prior research by 
elucidating the processes through which production activities affect product evaluation and how consumers relate to products. Moreover, the findings contribute to the extended-self literature by empirically measuring change in one dimension of person-object relationship, that is, identification with the product.

This paper also contributes to cross-cultural research. It reveals that self-construal influences when consumers' participation in the production process creates value. One insight gained is that, especially for consumers with independent self-construals, being involved in the production process does not enhance product evaluations unless the process is positive. Furthermore, consumers with interdependent self-construals evaluate products made with close others more favorably than other-made products (and as favorably as self-made products), whereas this tends not to be the case for consumers with independent selfconstruals.

For managers, the findings suggest that marketing stimuli (e.g., ads, packaging, store displays) that enable consumers to imagine themselves participating in the production process of a customized product may result in enhanced product evaluations. For example, advertisers may, instead of showing another person making the product, create ads that enable the consumers to think of themselves making or modifying the product and thus enhance vicariously the consumer's experience.

In Study 2, the production process (painting the white t-shirt) consisted of only minimally modifying the product. Results indicated that even being involved in such a small modification of the product enhanced product evaluations. Therefore, having consumers assemble even a limited number of final parts, for instance, may increase product evaluation and result in a stronger connection between the consumer and the product. For example, a company that markets custom-made products (e.g., Porsche making custom-made automobiles) might allow consumers to work on the configuration of the final product purchased online or to even customize the products at the retail store or factory with one's own labor and input to a certain extent to strengthen identification with the product.

In addition, results reveal that even negative production processes may enhance evaluation of selfmade products if the consumer has an interdependent self-construal. Hence, priming interdependent self-construal through packaging communication or ads may ensure that the self-production process creates value even when the company does not have full control over the valence of the process emotions.

Furthermore, as Study 3 findings suggest, if a product can be built or customized with two or more people, then priming interdependent self-construal may increase the value created through the production process. In line with this, advertisers may draw attention to the fact that a product might be built with close others in an interdependent culture (e.g., East Asia) but may be built individually (requires only one person to build) in an independent-based culture (e.g., United States).

\section{LIMITATIONS AND FUTURE RESEARCH}

Both hypothetical stories and an actual production situation have been used to investigate how the process valence affects the evaluation of the outcome product. However, the strength of emotions that the participants experience in a laboratory setting may be limited. Therefore, the findings may only apply to the experience of mild to moderate emotions. Consumers may change their evaluations of self-made products more when the process is extremely painful or unpleasant. Nevertheless, the experience of mild or moderate emotions represents many everyday consumption situations, and therefore the findings may be applicable to many consumption situations in everyday life.

Additionally, only valence of emotions that affect the value created due to production activities have been investigated. An important next step would be to investigate how other dimensions of emotions, such as differing attributions of agency or responsibility and control, affect evaluation of self-made products.

Another line of research might look at how product type affects whether one identifies with the products in the process of production. Product type may affect how much a particular domain is central to one's selfidentity. It is expected that, as the centrality of the domain increases, so would the likelihood that an individual will identify with a self-made product. A product that is highly central to one's of self-identity may result in stronger reactions when consumers are involved in the production or modification of the product.

Moreover, only one process, identification with the product, has been investigated to explain how participation in the production process creates value for consumers. Additional research is needed to investigate whether there are other factors (e.g., creativity, flow, need for uniqueness, need for control) that drive the value derived from being involved in the production process.

\section{REFERENCES}

Aaker, J. L., \& Schmitt, B. (2001). Culture-dependent assimilation and differentiation of the self: Preferences for consumption symbols in the United States and China. Journal of Cross-Cultural Psychology, 32, 561-576.

Aaker, J. L., \& Williams, P. (1998). Empathy versus pride: The influence of emotional appeals across cultures. Journal of Consumer Research, 25, 241-261.

Bagozzi, R. P., \& Dholakia, U. M. (2006). Antecedents and purchase consequences of customer participation in small group brand communities. International Journal of Research in Marketing, 23, 45-61.

Bagozzi R. P., Verbeke, W., \& Gavino, J. C. (2003). Culture moderates the self-regulation of shame and its effects on performance: The case of salespersons in the Netherlands 
and the Philippines. Journal of Applied Psychology, 88, 219-233.

Belk, R. W. (1988). Possessions and the extended self. Journal of Consumer Research, 15, 139-168.

Bendapudi, N., \& Leone, R. P. (2003). Psychological implications of customer participation in co-production. Journal of Marketing, 67, 14-28.

Bergami, M., \& Bagozzi, R.P. (2000). Self-categorization, affective commitment and group self-esteem as distinct aspects of social identity in the organization. British Psychological Society, 39, 555-577.

Campbell, D. T. (1963). Social attitudes and other acquired behavioral dispositions. In S. Koch (Eds.), Psychology: A study of a science (Vol. 6, pp. 94-172). New York: McGrawHill.

Campbell, J. D. (1986). Similarity and uniqueness: The effects of attribute type, relevance, and individual differences in self-esteem and depression. Journal of Personality and Social Psychology, 50, 281-294.

Chen, M., \& Bargh, J. A. (1999). Consequences of automatic evaluation: Immediate behavioral predispositions to approach or avoid the stimulus. Personality and Social Psychology Bulletin, 25, 215-224.

Ekman, P. (1984). Expression and the nature of emotion. In K. R. Scherer \& P. Ekman (Eds.), Approaches to Emotion (pp. 319-344). Hillsdale, NJ: Erlbaum.

Etgar, M. (2008). A descriptive model of the consumer coproduction process. Journal of the Academy of Marketing Science, 36, 97-108.

Forgas, J. P. (2003). Affective influences on attitudes and judgments. In R.J. Davidson, K.R. Scherer, \& H. H. Goldsmith (Eds.), Handbook of affective sciences (pp. 596-618). New York: Oxford University Press.

Fuchs, C., \& Schreier, M. (2011). Customer empowerment in new product development. Journal of Product Innovation Management, 28, 17-32.

Fuchs, C., Prandelli, E., \& Schreier, M. (2010). The psychological effects of empowerment strategies on consumers' product demand. Journal of Marketing, 74, 65-79.

Greenwald, A. G., \& Banaji, M. R. (1995). Implicit social cognition: Attitudes, self-esteem, and stereotypes. Psychological Review, 102, 4-27.

Greenwald, A. G., \& Leavitt, C. (1984). Audience Involvement in advertising: Four levels. Journal of Consumer Research, 11, 581-592.

Hayes, A. F. (2013). An introduction to mediation, moderation, and conditional process analysis: A regression-based approach. New York: Guilford Press.

Heine, S. J., \& Lehman, D. R. (1999). Culture, selfdiscrepancies, and self-satisfaction. Personality and Social Psychology Bulletin, 25, 915-925.

Heine, S. J., Kitayama, S., \& Lehman, D. R. (2001). Cultural differences in self-evaluation: Japanese readily accept negative self-relevant information. Journal of Cross-Cultural Psychology, 32, 434-443.

Krishna, A., Zhou, R., \& Zhang, S. (2008). The effect of selfconstrual on spatial judgments. Journal of Consumer Research, 35, 337-348.

Kuhn, M. H., \& McPartland, T. S. (1954). An empirical investigation of self-attitude. American Sociological Review, 19, 68-76.

Lee, A., Aaker, J. L., \& Gardner, W. L. (2000). The pleasures and pains of distinct self-construals: The role of interdependence in regulatory focus. Journal of Personality and Social Psychology, 78, 1122-1134.
Marks, G. (1984). Thinking one's abilities are unique and one's opinions are common. Personality and Social Psychology Bulletin, 10, 203-208.

Markus, H. R. (1977). Self-schemata and processing information about the self. Journal of Personality and Social Psychology, 35, 63-78.

Markus, H. R., \& Kitayama, S. (1991a). Cultural variation in the self concept. In G. R. Goethals \& J. Strauss (Eds.), Multidisciplinary perspectives on the self (pp. 18-48). New York: Springer-Verlag.

Markus, H. R., \& Kitayama, S. (1991b). Culture and the self: Implications for cognition, emotion, and motivation. Psychological Review, 98, 224-253.

Markus, H. R., \& Oyserman, D. (1989). Gender and thought: The role of the self-concept. In M. Crawford \& M. Gentry (Eds.), Gender and thought: Psychological perspectives (pp. 100-127). New York: Springer-Verlag.

Mittal, B. (1995). A comparative analysis of four scales of involvement. Psychology \& Marketing, 12, 663-682.

Mittal, B. (2006). I, me, and mine-how products become consumers' extended selves. Journal of Consumer Behavior, 5, $550-562$.

Mogilner, C., \& Aaker, J. (2009). "The Time vs. Money Effect": Shifting product attitudes and decisions through personal connection. Journal of Consumer Research, 36, 277-291.

Norton, M. I., Mochon, D., \& Ariely, D. (2012). The IKEA effect: When labor leads to love. Journal of Consumer Psychology, $22,453-460$

Olsen, S. O., \& Mai, H. T. X. (2013). Consumer participation: The case of home meal preparation. Psychology \& Marketing, 30, 1-11.

Petty, R. E., \& Cacioppo, J. T. (1986). Communication and persuasion: Central and peripheral routes to attitude change. New York: Springer-Verlag.

Pierce, J. L., Kostova, T., \& Dirks, K. T. (2003). The state of psychological ownership: Integrating and extending a century of research. Review of General Psychology, 7, 84107.

Prahalad, C. K., \& Ramaswamy, V. (2004). The future of competition: Co-creating unique value with customers. Boston, MA: Harvard Business School Press.

Reed, A., Aquino, K., \& Levy, E. (2007). Moral identity and judgments of charitable behaviors. Journal of Marketing, $71,178-193$

Russell, J. A. (1980). A circumplex model of affect. Journal of Personality and Social Psychology, 39, 1161-1178.

Russell, J. A., \& Mehrabian, A. (1978). Approach-avoidance and affiliation as functions of the emotion eliciting quality of an environment. Environment and Behavior, 10, 355387.

Sartre, J. P. (1943). Being and nothingness. A phenomenotogical essay on ontology. New York: Philosophical.

Seibt, B., Neumann, R., Nussinson, R., \& Strack, F. (2007). Movement direction or change in distance? Self and object related approach-avoidance motions. Journal of Experimental Social Psychology, 44, 713-720.

Smith, C. A., \& Ellsworth, P. C. (1985). Patterns of cognitive appraisal in emotion. Journal of Personality and Social Psychology, 48, 813-838.

Tesser, A., \& Paulhus, D. (1983). The definition of self: Private and public self-evaluation management strategies. Journal of Personality and Social Psychology, 44, 672-682.

Toffler, A. (1980). The third wave. New York: William Collins Sons and Co. Ltd.

Troye, S. V., \& Supphellen, M. (2011). Consumer participation in coproduction: "I Made It Myself" effects on consumers' 
sensory perceptions and evaluations of outcome and input product. Journal of Marketing, 76, 33-46.

Van Boven, L., \& Gilovich, T. (2003). To do or to have: That is the question. Journal of Personality and Social Psychology, 85, 1193-1202.

Vargo, S. L., \& Lusch, R. F. (2004). The four service marketing myths: Remnants of a goods-based, manufacturing model. Journal of Service Research, 6, 324-335.

Weinstein, N. D. (1989). Effects of personal experience on selfprotective behavior. Psychological Bulletin, 105, 31-50.

White, K., \& Argo, J. J. (2009). Social identity threat and consumer preferences. Journal of Consumer Psychology, 19, 313-325.

White, K., Argo, J. J., \& Sengupta, J. (2012). Dissociative versus associative responses to social identity threat: The role of self-construal. Journal of Consumer Research, 39, 704719 .

Williams, C. C. (2008). Re-thinking the motives of do-ityourself (DIY) consumers. International Review of Retail, Distribution and Consumer Research, 18, 311-323.

Wolf, M., \& McQuitty, S. (2011). Understanding the doit-yourself consumer: DIY motivations and outcomes. Academy of Marketing Science Review, 1, 154-170.

Xie, C., Bagozzi, R. P., \& Troye, S. V. (2008). Trying to prosume: Toward a theory of consumers as co-creators of value. Journal of the Academy of Marketing Science, 36, 109-122.

Xie, C., Bagozzi, R. P., \& Ostli, J. (2013). Cognitive, Emotional, and Sociocultural Processes in Consumption. Psychology \& Marketing, 30, 12-25.

Zaichkowsky, J. L. (1985). Measuring the involvement construct. Journal of Consumer Research, 12, 341-352.

The authors thankfully acknowledge the helpful input of Michael Norton and Jeffrey Sanchez-Burks on an earlier draft of the manuscript.

Correspondence regarding this article should be sent to: Sukriye Sinem Atakan, Assistant Prof. Graduate School of Business, Ozyegin University, Istanbul, Turkey (sinem.atakan@ozyegin.edu.tr).

\section{APPENDIX A: STUDY 1 STIMULI}

\section{Negative Process Condition}

Imagine that in a pottery class you (another participant in this experiment) spent 40 minutes making a ceramic coffee mug from clay. You (the participant) found the process and the activity of making the clay very unpleasant and you (s/he was) were very unhappy while making the mug. As you (s/he) sculpted the mug, the clay felt clumpy and gross. You (s/he) disliked the smell of clay and the feeling of wet clay in your (his/her) hands gave you (her/him) the chills. Your hands were irritated and you (S/he) had to keep washing your (his/her) hands to get the dirt off of them. You felt the smell of the clay was even on your (his/her) clothes later. The whole process was irritating and dissatisfying. In the end, the mug came out looking like below.

\section{Positive Process Condition}

Imagine that in a pottery class you (another participant in this experiment) spent 40 minutes making a ceramic coffee mug from clay. You (the participant) found the process and the activity of making the mug very pleasant and you (s/he was) were very happy while making the mug. As you (s/he) sculpted the mug, the clay was smooth and soothing. You (S/he) loved the smell of clay and the feeling of wet clay in your (his/her) hands made you (her/him) relaxed and satisfied. Your hands felt warm and you (s/he) just wanted to let the clay dry on your (his/her) hands rather than washing it off. You relished the smell of the clay even on your clothes later. The whole process was fun and satisfying. In the end, the mug came out looking like below. 


\section{APPENDIX B: STUDY 2 ROOM PICTURES}

\section{Positive Process Condition}

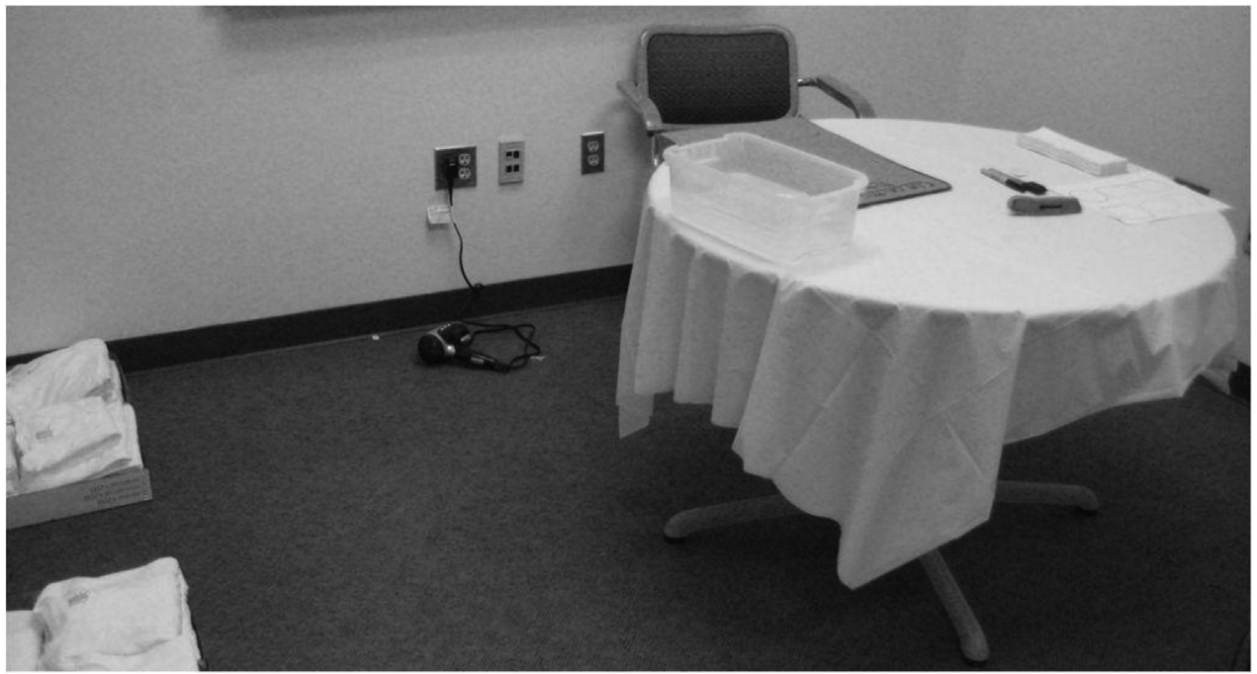

\section{Negative Process Condition}

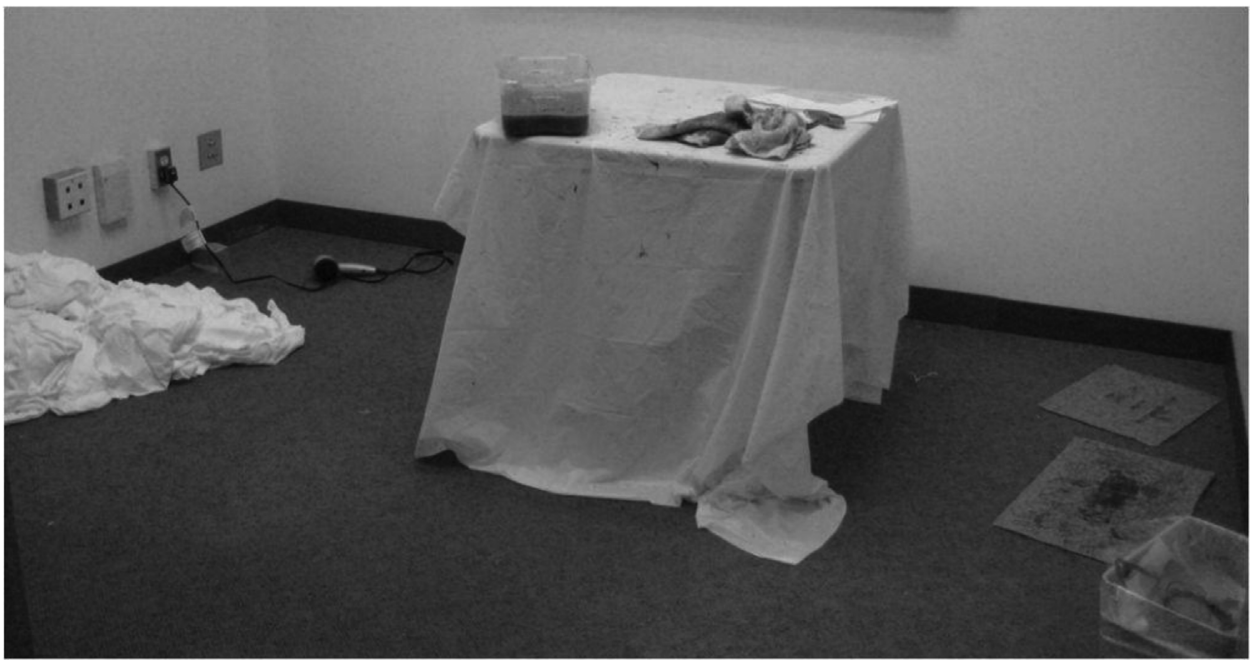

\section{APPENDIX C: STUDY 3 STIMULI}

\section{Group Condition}

Please type the initials or nicknames of your two friends and indicate how long you have known him/her:

Friend 1 I have known him/her I have known him/her years.

Friend 2 years.

\section{Group/Self/Other Conditions}

T-Shirt Design Experience. Please try to imagine yourself in the following scenario as vividly as possible. You have a white t-shirt that you/you/was designed and made with two of your friends whom you just identified / yourself / by a complete stranger that you have never met. You/You/this person spent almost six hours together working on the t-shirt, and everyone actually found the whole process and the activity very pleasant (unpleasant). Everybody/you/s/he was quite happy (unhappy) and relaxed (stressed out) while trying to make the t-shirt.

First, you and your friends/you/the stranger decided to apply an iron-on transfer to the front of white t-shirt. The white t-shirt felt like a friendly, inviting canvas (the white t-shirt felt like a daunting canvas). You/you/s/he had a photo you/you/s/he took last summer, and you/you/s/he printed it on a transfer paper. Next, one of you/you/s / he placed the t-shirt on 
a hard surface and the photo onto the t-shirt. Then, you/you/s/he set the iron at a high temperature because you/you/s/he did not want the image to peel off easily later on. You / you/s / he carefully checked the iron temperature and started to iron the outside edges first and then moved toward the center. You/you/s/he was calm and comfortable as you finished ironing the photo and peeled the transfer paper off the front of the t-shirt. (However, you/you/s/he burned yourself/yourself/himself/herself when you/you/s/he tried to check whether the iron was hot enough. You/You/ s/ he got angry and tense as your/your/his/her fingers got red and started burning. You/you/s/he finished ironing the photo and peeled the transfer paper off the front of the t-shirt.)

Next, as a group, you/you/s/he started to work on the back of the t-shirt and you / you / s / he decided to use computer software to design the image this time. Together, you / you/s / he tried to find the right shapes and prints that would complement each other, and decided to use your/your/his/her favorite quotes and sayings. You/you/s/he played with the letters and placed the text into the design. It required a good sense of layout and composition. You and your friends/you/s/he had literally never designed an image in your lives/your life / his / her life before, and fortunately (unfortunately) it felt fun and inspiring (irritating and boring). All of $y o u / y o u / s / h e$ was very entertained (annoyed) in the process because you/you/s/he got the chance to play and experiment with the shapes and colors as you/you/s/he wished (because you/you/s/he was very frustrated with all the shapes and colors that you/you/s/he had to edit). Figuring out the final design involved some trial and error but it was the one of the most enjoyable (and it turned out to be one of the most wearisome) things all of you / you/s/he have done in a while!

Finally, you and your friends / you / s / he went to (had the hassle of going to) a print shop to have the design printed on the back of the t-shirt. You/you/s/he did not need to wait at the shop and it was done quickly (you/you/s/he had to wait at the shop forever since they had to finish a big order before yours). The whole process of making the t-shirt gave everybody/you/him/her a thrill (headache)! It felt rewarding (silly) to spend time and energy on it, and for all of you / him/her the overall process of making the t-shirt was surprisingly fun (sucked)! 